large distances this varies with azimuth by nearly 5 seconds when geographical co-ordinates are used, and introduces a large systematic error into southern epicentres if it is not allowed for. Bullen and I found on determining epicentres from the nearer stations $^{3}$, that southern earthquakes habitually showed negative residuals of about $-4 s$. at great distances, and attributed these to slight focal depth; it appears now, however, that they were entirely due to the ellipticity. The great majority of Pacific earthquakes can be taken as having foci at an inappreciable depth.

The law of error in seismological observations seems to be practically the normal law with a uniform distribution superposed. The standard error corresponding to the normal part is found to be $2 \cdot 0 s$. both for the good and for the intermediate stations; the difference between them is not in the accuracy of the normal observations, but in the frequency of defective ones, usually attributable to microseisms and weak beginnings.

There appears to be a slight difference between Pacific and continental travel times, amounting to about $2 s$. for $P$. between $5^{\circ}$ and $50^{\circ}$ out of a total time of 8 minutes, and indicating a higher velocity below the Pacific. This might be expected if there has been more cooling below the oceans than under the continents. Larger differences have been claimed previously, of the order of 10 per cent, but these are rendered impossible by the observations now available.

\section{St. John's College,} Cambridge. July 13.

1 Mon. Not. Roy. Ast. Soc., Geophys. Supp., 3, 423-443 (1936).

${ }^{2}$ Mon. Not. Roy. Ast. Soe., Geophys. Supp., 4, 143-157 (1937).

${ }^{3}$ Bur. Centr. Seism. Trav. Sci., 11 (1935).

\section{Magnetic Quality of Nickel Wire as Influenced by the Surface}

IN a previous note ${ }^{1}$, test data were given which showed the very large increase of magnetic permeability of nickel wire which could be obtained by applying to the surface a thin skin of copper and allowing it to soak for some hours at a sufficiently high temperature.

The influence of the surface of a wire on its magnetic quality has been examined further as follows :

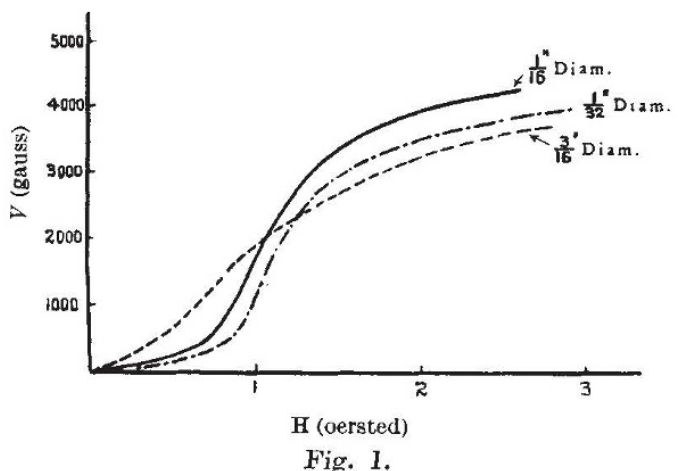

Commercially pure nickel wires of diameters $1 / 32$ in., $1 / 16$ in., and $3 / 16$ in. respectively, were heated in an atmosphere of hydrogen for a sufficiently long time to reach a condition of maximum permeability in each case. After having cooled slowly to room temperature, the magnetization curves for each wire was obtained and these curves are shown in Fig. 1.

It will be seen that the initial portion of the curve for the 1/32-in. diameter wire is very flat and this part of the curve becomes steeper as the diameter increases. That is to say, the greater the ratio of surface perimeter to cross-sectional area, the less easily is the wire magnetized for low values of $H$. In other words, the magnetic characteristic of the wire is intimately dependent upon the diameter. The tests have also shown that the remanence and the coercive force are both considerably greater for the wire of $1 / 32$ in. diameter than for the other wires.

These results have important theoretical and practical implications, which will be considered in a paper which is now in course of preparation for publication elsewhere.

Department of Electrical Engineering,

$$
\text { T. F. WALL. }
$$

The University,

Sheffield, 1.

July 6.

${ }^{1}$ NAture, 139, 928 (May 29, 1937).

\section{Darkening of some Commercial Titanium Dioxide in Daylight}

DURING an investigation of the discoloration by light of some ceramic glazes containing titanium compounds and of the subsequent fading of the discoloration when the glaze is kept in the dark ${ }^{1}$, it was observed that certain varieties of commercial titanium dioxide are themselves affected by daylight. These varieties pass from their normal creamy colour to a deeper yellow-brown tint on exposure. The original colour is restored in the dark. This property of some commercial titania appears to have escaped notice in Great Britain, though $\mathrm{Lee}^{2}$, in his study of reversible photo-sensitivity in hackmanite, draws an analogy with a certain brand of titania which showed the property mentioned in this letter.

The samples of titanium dioxide studied were stored in ordinary glass vessels exposed to daylight transmitted through the glass of the laboratory windows. The darkening of the titania, observed against samples kept in the dark, was apparent after only three hours in some instances. The effect is shown by those particles or perhaps portions of particle-surfaces adjacent to the illuminated glass wall of the container. Hence it is important that the powdered titania should move as little as possible during illumination if the darkening is to be obvious.

No experiments were made with the photo-sensitive brands of titania as components of paints or pig. ments, but they conferred similar properties on some ceramic glazes containing them. However, ceramic glazes darkening in light were made from titania and from rutile where these materials themselves showed no apparent darkening.

A definite explanation of the darkening of titania cannot be cited, though it appears likely that the colours shown by the exposed and by the unexposed material mark the establishment of an equilibrium condition of either a chemical or a physical nature. A chemical explanation might involve the oxidation of ferrous iron present in the titania to a more deeply coloured ferric compound (cf. the possible 